\title{
Study on the Mechanism of Cathepsin L on the Activation of M1 Macrophages in Sepsis-induced Acute Renal Injury
}

YAFANG. HU, LIANGQI. GUO'1, KAIJUN. GAO ${ }^{1}$ AND MOUCHENG. ZHANG ${ }^{2 *}$

Department of Anesthesiology, Ningbo First Hospital, Ningbo, Zhejiang 315000, ${ }^{1}$ Medicine School of, Ningbo University, Ningbo, Zhejiang 315000, '2Department of Gastrointestinal Surgery, Ningbo First Hospital, Ningbo, Zhejiang 315000, China

Hu et al.: Study on the mechanism of Cathepsin $L$ in sepsis-induced acute renal injury

\begin{abstract}
The purpose of this study was to explore the mechanism of cathepsin $L$ and M1 macrophages in acute renal injury induced by sepsis. The sepsis model of male mice was established by cecal ligation puncture or sham operation, and the biochemical and histological renal damage in mice was evaluated. At the same time, infiltration of macrophages was detected by immunohistochemistry. The expression of cathepsin L-inducible nitric oxide synthase and argininase 1 messenger ribonucleic acid was analysed by reverse transcription polymerase chain reaction. Western blotting was used to detect the expression level of cathepsin $L$. High level of cathepsin $L$ was detected $24 \mathrm{~h}$ after sepsis-induced acute renal injury. The results of the western blot assay showed that cathepsin $L$ inhibitors successfully inhibited the expression of cathepsin $L$ during the sepsis-induced acute renal injury. After the establishment of the septic-induced acute renal injury model, cathepsin $L$ inhibitors alleviated the renal injury, while M1 macrophage activation was significantly inhibited. In sepsis-induced acute renal injury, cathepsin $L$ plays an important role in related initial inflammatory response by activating M1 macrophages.
\end{abstract}

Key words: Cathepsin L, sepsis, acute renal injury, rat model

Acute renal injury (ARI) is a common complication in patients with sepsis, with high morbidity and mortality ${ }^{[1]}$.
Sepsis is one of the most common causes of ARI ${ }^{[2]}$. At present, an animal model of sepsis has been developed, 
and it has been proved that the pathogenesis of ARI was caused by inflammatory cell infiltration, renal endothelial cell dysfunction, renal hemodynamic changes and nephrocyteapoptosis ${ }^{[3]}$. Sepsis-induced ARI is caused by a variety of mechanisms, including vascular leakage/hypoperfusion, local renal tubular inflammation and cell cycle arrest ${ }^{[4]}$. Among these factors, obvious tissue inflammation in the kidney seems to be the key factor in sepsis-induced ARI ${ }^{[5]}$. Recent studies have shown that the innate immune and inflammatory signalling pathways were involved in the pathogenesis of septic ARI, which begins with immune cell infiltration from the kidney a few hours after injury and is essential for kidney regeneration ${ }^{[6,7]}$. Macrophages are the most common white blood cells involved in the process of renal injury, which plays different roles in different stages of renal injury ${ }^{[8]}$. Due to the difference in immune microenvironment, macrophages have different functions in different periods. In the early stage of sepsis, macrophages undergo M1 differentiation, leading to the production of inflammatory factors and $A R I^{[9]}$. M1 macrophages upregulate the expression of proinflammatory factors, including inducible nitric oxide synthase (iNOS) and tumour necrosis factor- $\alpha$ (TNF- $\alpha$ ) and increase the production of reactive oxygen and nitrogen ${ }^{[10-12]}$. In contrast, antiinflammatory M2 macrophages upregulated the expression of arginase 1 (Arg-1), scavenger receptor, mannose receptors and found in inflammatory zone 1 (FIZZ1). INOS expression has been used as a marker of M1 response, while Arg-1 and FIZZ1 are classic inducers of M2 gene expression $^{[13]}$. Cathepsin L (CPL), a biomarker used to predict the occurrence and prognosis of severe sepsis and septic shock, has recently been proposed to participate in the pathophysiology of ARI. CPL is a chemokine for neutrophils, T cells and monocytes, and can enhance the cytokine release, phagocytosis and endothelial adhesion of monocytes ${ }^{[14,15]}$. The findings of Fisher et al. prove that CPL can cause renal inflammation and capillary leakage ${ }^{[16]}$. However, it is not clear whether CPL induces macrophages to promote inflammation. So far, there is no study on the relationship between CPL and M1 macrophages in sepsis-induced ARI. In order to further improve the understanding of sepsis-induced inflammation in ARI mice, sepsisinduced ARI mouse model was used to study the expression of CPL in vivo. It has been found that the inhibition of CPL expression by heparin injection can reduce the severity of the renal injury, and significantly inhibit the activation of M1 macrophages, and the expression of pro-inflammatory cytokines. Male mice
(6-8 w old) were fed in cages with a 12/12 h light/dark cycle, $25^{\circ}, 60 \%$ humidity environment, without specific pathogens. The cecal ligation and puncture (CLP) operation method described by Zhang was improved ${ }^{[15]}$. The main steps and methods are as follows, after laparotomy under isoflurane anesthesia, a 4-0 silk thread was tied $15 \mathrm{~mm}$ from the tip of the cecum. The cecum was punctured twice with a No.18 needle and gently squeezed to express a small amount of feces, and then pushed back into the abdomen. In sham-operated animals, the cecum is fixed but not ligated or punctured. The abdominal incision was divided into two layers with 6-0 nylon suture. After the operation, the animals were resuscitated by the subcutaneous injection of 40 $\mathrm{ml} / \mathrm{kg}$ sterile saline, and they can drink freely, but could not eat. During surgery, the body temperature is maintained at $37^{\circ}$. The animals were killed by neck dislocation after $72 \mathrm{~h}$, and the blood and kidney samples were collected and stored at $-80^{\circ}$ for further analysis. Mice were intraperitoneally injected with $0.4 \mu \mathrm{g} / \mathrm{kg}$ CPL inhibitor or phosphate buffer $12 \mathrm{~h}$ before sepsis to prepare liposomes.The tissue was fixed with $10 \%$ formalin and embedded in paraffin. The sections with a thickness of $4 \mu \mathrm{m}$ were stained by periodic acid-Schiff (PAS) reagent. Histological changes of cortical and OSOM were evaluated by quantitative measurement of tissue injury. Because the renal tubular injury is mainly vacuolization, the injury is defined as renal tubular vacuolar degeneration. The following criteria were used to estimate the degree of renal damage in 200-fold magnified images of more than 100 randomly selected renal tubules per animal. 0 , normal; 1 , renal tubules with an injury area less than $25 \% ; 2,25-50 \%$ of damaged renal tubules; $3,50-75 \%$ of injured renal tubules; $4,75-100 \%$ of damaged renal tubules. The sections were blindly graded by two pathologists to avoid histological analysis bias.RNA was extracted from frozen kidneys stored at $-80^{\circ}$ using standard procedures and treated with DNase I, and then reverse transcribed with $0.5 \mathrm{~g}$ total RNA. The reverse transcripts products were diluted 20 times for PCR, with the following primer pairs. iNOS-F, 5'-GAATTCCCAGCTCATCCG GT-3', iNOS-R, 5'GGTGCCCATGTACCAACCGGT-3', Arg-1-F, 5'-CCGCAGCATTAAGGAAAGC-3', Arg-1-R, 5'-CCCGTGGTCTCTCACACG-3', CPL-F, 5'-ACAACCTCAACGTCATCCTG G-3', CPL-R, 5'-GTCTTCATTGAGGGCGTTGC-3', $\beta$-actin-F, 5'-CAGTAACAGTCCGCCTAGAA-3', $\beta$-actin-R, 5'-GATTACTGCTCTGGCTCCTA-3'. Annealing temperature $(\mathrm{Tm}) 55^{\circ}, 30$ cycles, $\mathrm{PCR}$ products were 
separated on $2 \%$ agarose gel and stained with ethidium bromide. Renal tissue sections were stained with F4/80 and Fi67 by immunohistochemical staining. The kidney sections of $3 \mathrm{~mm}$ were dewaxing and rehydrated with alcohol of gradient concentration. The sections were immersed in $3 \%$ hydrogen peroxide for $10 \mathrm{~min}$ to block the activity of endogenous peroxidase, and then blocked in normal horse serum. Then, the sections were immersed in $0.1 \mathrm{M}$ citrate buffer ( $\mathrm{pH} \mathrm{6.0)}$ ) for $25 \mathrm{~min}$ and heated in an electric pressure cooking saucepan for 5 min for antigen repair. The sections were incubated with mouse CD68 monoclonal antibody (1:100, Abcam, USA) at $4^{\circ}$ overnight. The primary or secondary antibodies were omitted in the control experiment. Then, the sections were incubated with horse radish peroxidase (HRP) labelled goat antirabbit or rat antimouse secondary antibody (Acon Biotechnology Co., Ltd., Hangzhou) at room temperature for $1 \mathrm{~h}$. 3,3-diaminobenzidine tetrahydrochloride (DAB, Acon Biotechnology Co., Ltd., Hangzhou) was then dripped onto the sections to produce brown, and red dye with hematoxylin and pictomicrographs were taken with an Olympus camera. The whole cell extract was prepared. The protein sample was separated on $10 \%$ SDS-PAGE gel, transferred to PVDF membrane and blocked with $5 \%$ skimmed milk. Then, the membrane and the primary antibody were incubated overnight in $4^{\circ}$ and incubated further with the HRP labelled secondary antibody for $1.5 \mathrm{~h}$. Protein bands were detected by enhanced chemiluminescence (ECL) detection system (Pierce, Rockford, USA).The results were expressed as mean \pm SEM. Wilcoxon test is used to analyse the non-parametric data. The statistical comparison between the experimental groups was evaluated by Student's t-test and one-way ANOVA of SPSS 20.0. $\mathrm{p}<0.05$ was considered to be significant.

In order to detect the production of renal CPL in the sepsis-induced ARI, CLP was performed on mice which were sacrificed at different times (fig. 1). In shamoperated mice, the expression of CPL in the kidney is very low. However, in sepsis-induced ARI mice, the expression of CPL in the kidney increased significantly within $24 \mathrm{~h}$. In order to obtain more detailed evidence, qPCR was used to analyse the expression of CPL in the kidney and a high level of CPL was detected $24 \mathrm{~h}$ after the operation.Histological examination and renal tubular injury score (fig. 2) was evaluated $24 \mathrm{~h}$ after sepsis-induced ARI and extensive lesions were observed, including epithelial dilatation, flattening and swelling, loss of proximal renal tubular epithelial cells and vascular cast. The results showed that CPL increased renal damage and renal dysfunction during renal injury induced by sepsis.

Renal cortical tissue sections were stained with specific antibodies against mouse CD68 to evaluate the degree of macrophage infiltration during renal injury after CLP surgery (fig. 3). The sham-operated mice showed the smallest interstitial macrophage staining. However,
A

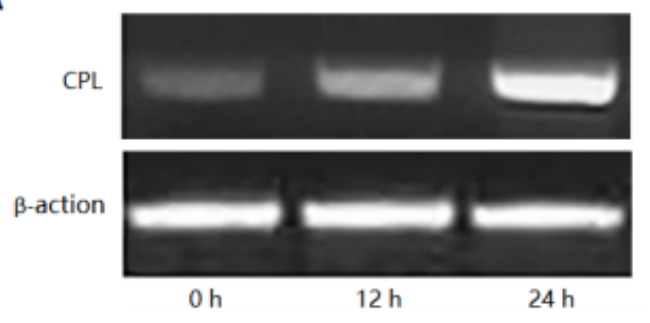

B

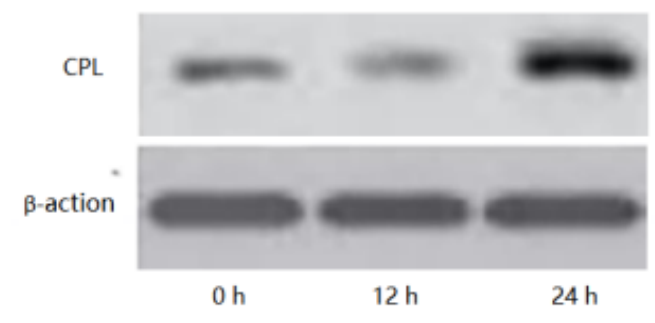

C

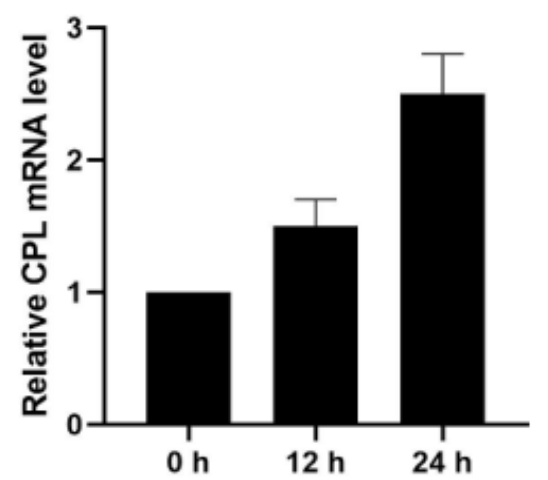

Fig. 1: CPL mRNA level after sepsis-induced ARI in mice 
mice with CLP surgery showed significant macrophage accumulation $24 \mathrm{~h}$ later. In the presence of CPL, the degree of macrophage infiltration in sepsis-induced ARI decreased significantly. Therefore, CPL induces macrophage activation and infiltration after sepsisinduced ARI. The expression of iNOS mRNA in M1 macrophages was higher than that in M2 macrophages, while the expression of Arg-1 and FIZZ1 mRNA increased in M2 macrophages. RT qPCR analysis was performed on mouse renal tissue sections to determine the expression of M1/M2 macrophage markers at different time points after CLP operation (fig. 4). The level of iNOS mRNA detected in the CLP and Control groups was higher than that in the CPL+CLP group. However, the expression of FIZZ1 and Arg-1 mRNA remained low in all three groups, indicating that CPL promoted the differentiation of M1 macrophages after sepsis-induced ARI.Sepsis-induced ARI is a common disease associated with high morbidity and mortality ${ }^{[16]}$. Macrophages are pleiotropia cells of the innate immune system, and their functions span host defense, cytotoxicity, clearance of apoptotic cells and promotion of tissue repair. Macrophages are also

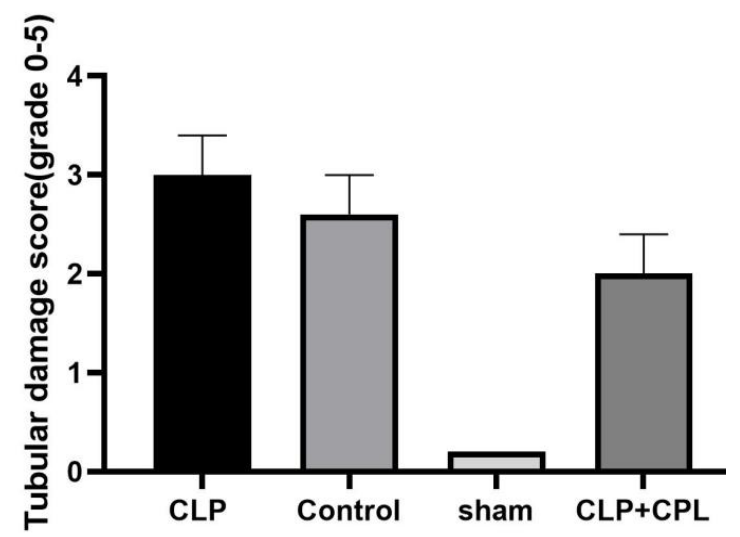

Fig. 2: Effects of CPL on histology and renal function in sepsis-induced ARI mice considered to be important mediators of renal injury in other experimental models of kidney disease, including transplantation, obstruction and glomerulonephritis ${ }^{[17]}$. Macrophages can be released from the bone marrow into the circulatory system and differentiate into resident macrophages to respond to certain stimuli. Inflammation is closely related to the activation of macrophages. M1 macrophages show pro-inflammatory activity, while M2 macrophages are involved in the resolution of inflammation ${ }^{[18,19]}$. According to their potential mechanism, M1 macrophages play a key role in antigen presentation, pro-inflammatory cytokine secretion and phagocytosis. The rationality of CPL as a marker of infectious organ dysfunction can be supported by its strong effect on the release of immune factors, immune cells and endothelial cells production in the early stage of infection, which may be the pathogenic factor of sepsis $^{[20,21]}$. In this study, mice were injected with CPL solution $12 \mathrm{~h}$ after sepsis-induced ARI was established. During the sepsis-induced ARI, the expression of CPL was still low. In the past decade, many studies have confirmed that CPL can act as a chemotaxis signal of macrophages and promote the accumulation of macrophages at the infected site ${ }^{[22]}$. CPL in endothelial tissue can induce monocytes to accumulate at the infected site through calcium-dependent channels. CPL can also activate monocytes and increase the phagocytosis of macrophages ${ }^{[23]}$. In this study, the expression of CPL increased significantly $24 \mathrm{~h}$ after the operation and decreased in the following $\mathrm{h}$, which is consistent with the following findings. The expression of CPL increased significantly in the early stage of sepsis-induced ARI. Previous studies have shown that $24 \mathrm{~h}$ after sepsis-induced ARI, there were a large number of M1 macrophages, so it was possible to determine the potential relationship between macrophages and CPL. Therefore, it can be speculated that CPL might play
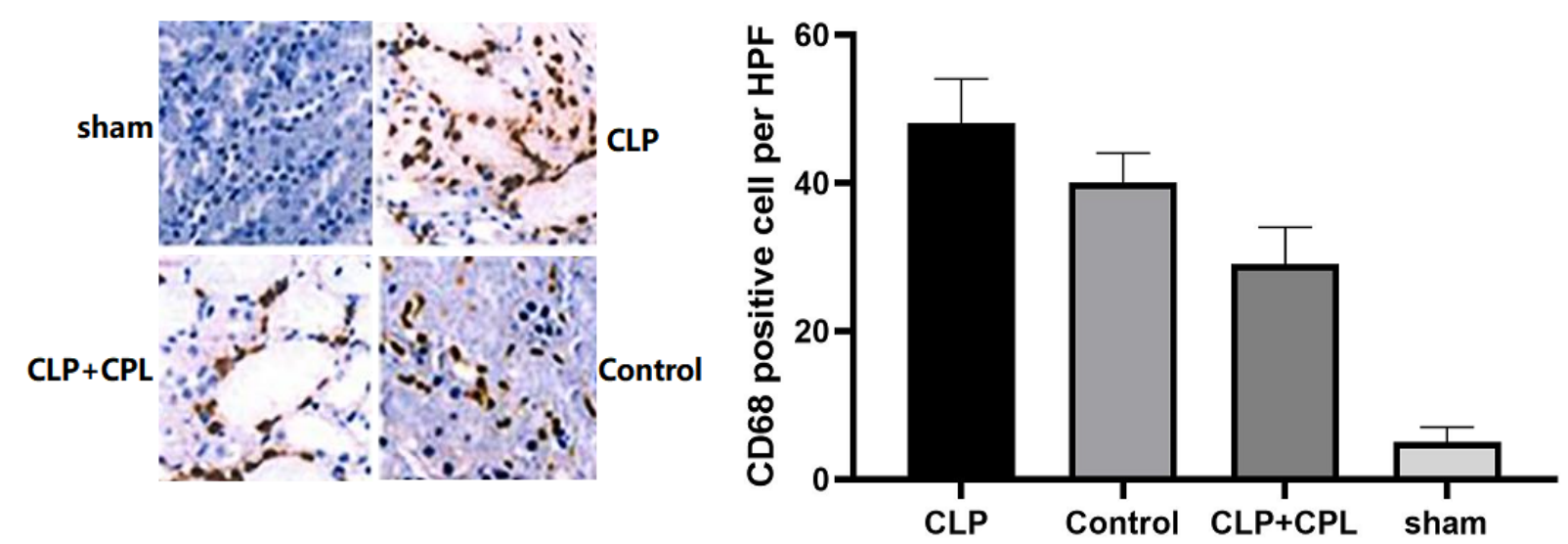

Fig. 3: Effect of CPL on macrophage infiltration in sepsis-induced ARI mice 


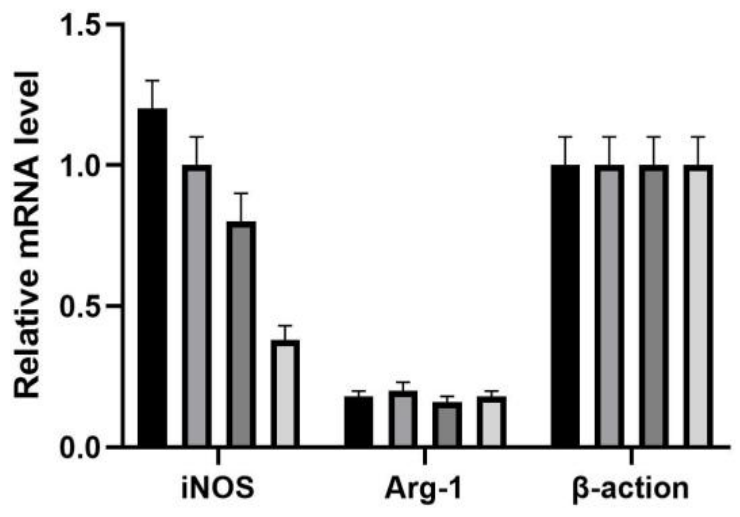

Fig. 4: Effect of CPL on differentiation of mouse M1 macrophages induced by sepsis-induced ARI

an important role in sepsis-induced ARI by activating M1 macrophages.Previous studies have shown that CPL induced renal tubular cell inflammation and renal endothelial cell loss in mice, which is a symptom of renal damage ${ }^{[24]}$. Studies have shown that CPL increased renal tubular injury and renal dysfunction and plays an important role in the initial inflammatory response associated with renal injury. After the establishment of sepsis-induced ARI, renal damage in sepsis-induced ARI can be reduced by inhibiting the expression of CPL in $24 \mathrm{~h}$. Interestingly, macrophage infiltration was also significantly reduced, indicating that renal tubular injury and macrophage infiltration could be reduced by inhibiting CPL. The potential role of CPL in M1 macrophage activation during septic-induced ARI was investigated as well as which subtypes of macrophages (M1 or M2) were mainly present in renal tissue sections of mice $24 \mathrm{~h}$ after sepsis-induced ARI. RT qPCR showed that the expression of iNOS increased in the kidneys of the 4 groups, but the expression of Arg-1 or FIZZ1 did not increase, indicating that CPL activated M1 macrophages in septic-induced ARI.The results showed that CPL played an important role in the initial inflammatory response related to sepsis-induced ARI, which might inhibit the secretion of some cellular inflammatory factors by activating M1 macrophages. Therefore, the strategy of limiting early macrophage infiltration or activation may be a new method for the prevention or treatment of ARI in patients with sepsis. However, the signal pathways involved in the activation mechanism of M1 macrophages needed to be further studied. Therefore, this research contributed to a better understanding of the complex events involved in sepsisinduced ARI, which is essential for the development of more effective treatment strategies.

\section{Authors contributions:}

Yafang $\mathrm{Hu}$ conceived and designed the experiments and gave an experimental guidance in the lab,

LiangqiGuo performed the experiments, KaijunGao analyzed the data and Moucheng Zhang wrote the paper.

\section{Acknowledgements:}

This work was supported by the Natural Science Foundation of Ningbo (No. 2019A610322).

\section{REFERENCES}

1. Schrier RW, Wang W. Acute renal failure and sepsis. N Engl J Med 2004; 351:159-69.

2. Klenzak J, and Himmelfarb J. Sepsis and the kidney. Crit Care Clin 2005;21:211-22.

3. Gomez H, Ince C, D De Backer, Pickkers P, Payen D, Hotchkiss $\mathrm{J}$, et al. A unified theory of sepsisinduced acute kidney injury: inflammation, microcirculatory dysfunction, bioenergetics, and the tubularcell adaptation to injury. Shock 2014;41:3-11.

4. Hotchkiss RS, Karl IE. The pathophysiology and treatment of sepsis. N Engl J Med 2003;348:138-50.

5. Ricci Z, Ronco C. Pathogenesis of acute kidney injury during sepsis. Curr Drug Targets 2009;10:1179-83.

6. Inoue T. M1 macrophage triggered by Mincle leads to a deterioration of acute kidney injury. Kidney Int 2017;91:526-9.

7. Lee S, Huen S, Nishio H, Nishio S, Lee HK, Choi BS, et al. Distinct macrophage phenotypes contribute to kidney injury and repair. J Am SocNephrol 2011;22:317-26.

8. Jo SK, Sung SA, Cho WY, Go KJ, Kim HK. Macrophages contribute to the initiation of ischaemic acute renal failure in rats. Nephrol Dial Transplant 2006;21:1231-9.

9. Sica A, Mantovani A. Macrophage plasticity and polarization: in vivo veritas. J Clin Invest 2012;122:787-95.

10. Nair MG, Du Y, Perrigoue JG, Zaph C, Taylor JJ, Goldschmidt $\mathrm{M}$, et al. Alternatively activated macrophage-derived RELM-a is a negative regulator of type 2 inflammation in the lung. $J$ Exp Med 2009;206:937-52.

11. Heo KS, Cushman HJ, Akaike M, Woo CH, Wang X, Qiu X, et al. ERK5 activation in macrophages promotes efferocytosis and inhibits atherosclerosis. Circulation 2014;130:180-91.

12. Obermajer N, Doljak B, Kos J. Cysteine cathepsins: regulators of antitumour immune response. Expert OpinBiolTher 2006;6:1295-309.

13. Boudreau F, Lussier CR, Mongrain S, Darsigny M, Drouin $\mathrm{JL}$, Doyon G, et al. Loss of cathepsin L activity promotes claudin-1 overexpression and intestinal neoplasia, FASEB J 2007;21:3853-65.

14. Zhang XL, Guo YF, Song ZX, Zhou M. Vitamin D prevents podocyte injury via regulation of macrophage M1/M2 phenotype in diabetic nephropathy rats. Endocrinology 2014; 155:4939-50.

15. Xu X, Greenland J, Baluk P, Adams A, Bose O, McDonald $\mathrm{DM}$, et al. Cathepsin L protects mice from mycoplasmal infection and is essential for airway lymphangiogenesis, Am J Respir Cell MolBiol 2013;49:437-44.

16. Zarjou A, Agarwal A. Sepsis and acute kidney injury. J Am SocNephrol 2011;22:999-1006.

17. Chang A, Ko K, Clark MR. The emerging role of the 
inflammasome in kidney diseases. CurrOpinNephrolHypertens 2014;23:204-10.

18. Gordon S, Plüddemann A, Martinez Estrada F. Macrophage heterogeneity in tissues: phenotypic diversity and functions. Immunol Rev 2014; 262:35-55.

19. Lundie RJ, Webb LM, Marley AK, Phythian-Adams AT, Cook PC, Jackson-Jones LH, et al. A central role for hepatic conventional dendritic cells in supporting Th2 responses during helminth infection. Immunol Cell Biol 2016;94:400-10.

20. Wille, A. Gerber, A. Heimburg, A. Reisenauer, C. Peters, P. Saftig, et al. Cathepsin L is involved in cathepsin D processing and regulation of apoptosis in A549 human lung epithelial cells. BiolChem 2004;385:665-70.

21. Watzke J, Schirmer K, Scholz S. Bacterial lipopolysaccharides induce genes involved in the innate immune response in embryos of the zebrafish (Daniorerio). Fish Shellfish Immunol 2007;23:901-905.
22. Nya EJ, Austin B. Use of bacterial lipopolysaccharide (LPS) as an immunostimulant for the control of Aeromonashydrophila infections in rainbow trout Oncorhynchusmykiss (Walbaum). J ApplMicrobiol 2010;108:686-94.

23. Wang R, Song L, Su B, Zhao H, Zhang D, Peatman E, et al. Mucosal expression signatures of two Cathepsin L in channel catfish (Ictaluruspunctatus) following bacterial challenge, Fish Shellfish Immunol 2015;47:582-9.

This is an open access article distributed under the terms of the Creative Commons Attribution-NonCommercial-ShareAlike 3.0 License, which allows others to remix, tweak, and build upon the work non-commercially, as long as the author is credited and the new creations are licensed under the identical terms

This article was originally published in a special
issue, "Biomedical Research in Healthcare Setting"
Indian J Pharm Sci 2020:82(3)Spl issue5;102-107

